\title{
Measles-containing vaccination rates in southern Alberta
}

\author{
Matkin $A^{1}$, Simmonds $K^{2}$ and Suttorp $\mathrm{V}^{3^{*}}$ \\ Faculty of Health Sciences, University of Lethbridge, Lethbridge, AB \\ 2 Surveillance and Assessment Branch, Alberta Health, Edmonton, AB \\ 3 Population and Public Health, Alberta Health Services, Lethbridge, AB \\ * Corresponding author: vivien.suttorp@albertahealthservices.ca
}

\begin{abstract}
Background: Southern Alberta is home to many unique homogeneous communities that typically educate their children in private schools. A number of these communities do not promote immunization as a preventive public health measure, although the reasons behind this vary. People within these communities keep themselves somewhat secluded from other populations and thus do not benefit from overall herd immunity. This has led to frequent outbreaks of vaccine-preventable diseases in private schools affiliated with these homogeneous religious communities.
\end{abstract}

Objective: To report on low immunization rates of measles, mumps, rubella (MMR) and MMR-varicella in southern Alberta communities and schools and to compare the epidemiology of immunization rates in certain vulnerable communities with those of same-age cohorts in South Zone communities.

Methods: The analysis includes immunization data at the individual level submitted to the provincial immunization repository, Immunization and Adverse Reactions to Immunization, and the Alberta Health Services Meditech module between January 1, 2013, and June 30, 2013.

Results: Heterogeneity of immunization status was found among communities and among schools. The status of two year old children up to date on immunizations ranged from $46.6 \%$ in Fort Macleod to $71.9 \%$ in Oyen, with a mean of 57.3 children in every 100 up to date. By age seven, the mean percentage of immunized children in southern Alberta was $77.6 \%$, ranging from $57.8 \%$ in Picture Butte to $94.6 \%$ in Oyen. Immunization status among schools ranged from $17 \%$ to $100 \%$, with a mean of $89.3 \%$ of children fully immunized and a median of $91 \%$ immunized.

Conclusion: There is heterogeneity of immunization uptake for childhood measles-containing vaccine by community and by school in southern Alberta. This study highlights that the location of the school may not align with geographic community as it pertains to immunization rates. Analysis of childhood immunization data at both community and school level is important in understanding the risks of vaccine-preventable illness spread in a given geographic region, such as Alberta South Zone. Data from this study can be used to inform specific interventions required to improve immunization coverage rates in these unique homogeneous cultural communities and their respective schools, and to decrease the risk of measles transmission in Southern Alberta.

\section{Introduction}

Measles is a highly communicable disease caused by the direct transfer of the measles virus by respiratory droplets or small-particle aerosols to human hosts. Particles remain suspended in air for up to two hours, and transmission of the virus is best prevented by active immunization with measles vaccine (1). In order to achieve successful herd immunity for measles disease, Alberta Health has set the target of $98 \%$ for children of two years of age to have received one dose of measles-containing vaccine, and $99 \%$ of children by 7 years to have received two doses of measles-containing vaccine (2). Preventing transmission remains dependent on a number of factors, such as a highly immunogenic vaccine; random mixing of a heterogeneous population; and consistent vaccination coverage among groups (3). The measles vaccine is highly effective, a single dose conferring $85 \%$ $95 \%$ immunity and a booster dose raising the effectiveness to almost $100 \%$ (4). Within a given population, some 
individuals will choose not to immunize. Random mixing refers to the importance of these non-immune individuals being incorporated into the portion of the population that is immunized, where they can be protected from acquiring disease through herd immunity. Herd immunity protects communities from measles outbreak by preventing the rapid spread of the virus. Although the number of children infected with and dying from measles per annum has decreased drastically since the routine administration of the measles vaccine, the disease still remains a public health problem and is returning to countries from which it was once thought to be eliminated or nearly eliminated (1,5-7). Strong religious or cultural beliefs against immunization, increasing travel across borders and continents, homogeneous subpopulations with insufficient vaccination coverage and lack of information or misinformation about vaccine safety are contributing to the re-emergence of measles $(3,7)$.

The most southern part of Alberta, the area south of Calgary, is one of five geographic areas within Alberta where health care is provided through Alberta Health Services (AHS). There are 17 public health offices (PHOs) in South Zone where children receive immunizations. Within South Zone there are various socially isolated communities with low immunization uptake for varying reasons (8). The largest of these communities is represented by the Netherlands Reformed Congregation, Low German Speaking Mennonite communities, and some Hutterite colonies. Each of them shares unique religious and/or cultural beliefs, and they are highly interconnected within their own community, resulting in minimal random mixing with the rest of the population in their geographic area. The majority of children from these communities are transported by school bus to private schools or are home-schooled, and participate in extracurricular activities such as sports and church primarily within their tight-knit social networks. Individuals have strong ties to countries such as the Netherlands, Mexico and South America, as well as other similar cultural settlements across North America. Travel to related communities with low immunization rates poses a risk of importation of vaccine-preventable disease to southern Alberta and potential high risk of spread. Although within some of these conservative religious communities there are individuals and families beginning to accept immunization, many still do not immunize their children. Cultural norms and expectations make it challenging for individuals to make informed decisions about immunization (810).

Historically in southern Alberta, there are frequent vaccine-preventable disease outbreaks. For example, over the past 20 years pertussis outbreaks have occurred every 3 to 5 years. The last large pertussis outbreaks in this area were in 2009 and 2012, in different religious communities. Analysis of the 2009 outbreak demonstrated that the outbreak originated in one of the private schools with very low immunization rates, and that there was ongoing transmission over an 11 month period within this school. Two months into the outbreak, pertussis illness spread to public schools in the geographic communities where children from the index school reside. Because of immunization rates that were closer to reaching herd immunity targets in public community schools, there was minimal transmission in these schools.

The objective of this article is to report on low MMR/MMR-V immunization rates in southern Alberta communities and schools, and to compare the epidemiology of immunization rates in certain vulnerable communities with that of similar age cohorts in the general population. Analysis of immunization rates in the geographic areas serviced by local PHOs are conducted to determine which areas have the highest and lowest uptake and the conclusions will inform future efforts to determine the best methods of intervention and education.

\section{Methods}

Measles, mumps, rubella and, more recently, varicella immunization in Canada is provided through a single vaccine (MMR or MMR-V) delivered in two doses (11). In Alberta, all childhood immunization is delivered by Alberta Health Services Public Health at public health offices and in school settings, with immunization policy and directives provided by Alberta Health. According to the Alberta Health routine immunization schedule, children in Alberta receive their first dose at 1 year and their second at 4-6 years (11). Individual-level data on immunizations in Alberta South Zone are entered at the point of care into the Meditech immunization module. Meditech data are submitted to the Alberta Health provincial repository, known as Imm/ARI (Immunization and Adverse Reactions to Immunization). In this study, community immunization data were obtained from the Imm/Ari database using postal codes, and school-level data were obtained from Meditech. Only children attending public and private schools were included; home-schooled children were not captured in the analysis. Schools were grouped by postal code into the PHOs used to analyze community immunization data. Two year 
old children with zero doses of vaccine were defined as unimmunized, and those with one dose were considered up to date on their immunizations. Seven year old children with zero doses (unimmunized) were considered as potentially those whose parents refused immunization; children with a single dose were categorized as partially immune; and those with two doses (up to date) were considered fully immunized.

Immunization data not accurately captured in this analysis were data for children who had been immunized in other jurisdictions in Alberta or out of province and who had not yet had their records updated by public health. These included First Nations children who received their immunizations through federally administered programs. Given this, immunization data for the towns of Standoff and Brocket, within two First Nations communities, were not included in the analysis. Additionally, immunization rates in Cardston need to be interpreted with caution as the postal codes for Cardston include areas where children receive immunization through the federal program.

\section{Data analysis}

Immunization rates per 100 population at the community level were calculated using available denominators and demographic data from Imm/ARI. Population data by PHO for children who were two and children who were seven by June 30, 2013, were used. The ages two and seven years were chosen on the basis of the routine immunization schedule (9); these ages allow a year of leeway for children to receive their doses on schedule. We compared number of doses of vaccine that children had received by age two (zero doses or at least one dose) and by age seven (zero doses, one dose or at least two doses) in each PHO catchment area. The number of children for each cohort (age 2 and 7 years) with zero, one or two doses was included in the numerator and the total number of children in that age group was the denominator. The quotient was multiplied by 100 to obtain incidence per 100 children. Immunization rates by school were similarly calculated using data from the Meditech module for children attending public and private schools who were seven by June 30, 2013.

\section{Results}

Findings indicate that $42.8 \%$ of two year old children in the AHS South Zone were unimmunized and $57.3 \%$ were up to date on their immunizations as of June 30,2013 . At that time, $14.3 \%$ of seven year old children were unimmunized, $8.1 \%$ were partially immunized, and $77.6 \%$ were fully immunized. Immunization rates by age and community are shown in Figures 1 and 2.

Figure 1. Immunization status of children by age two and by age seven in AHS South Zone

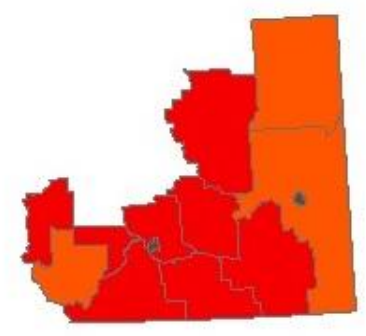

(a) Measles Coverage, one dose: 30 June 2013, by age 2
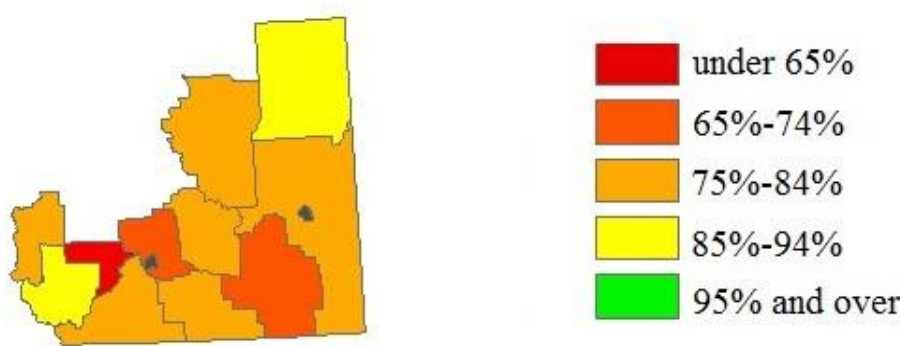

(b) Measles Coverage, two doses, 30 June 2013 , by age 7 
Figure 2. Immunization incidence rates per 100 eligible population by age seven in AHS South Zone Public Health Office catchment area

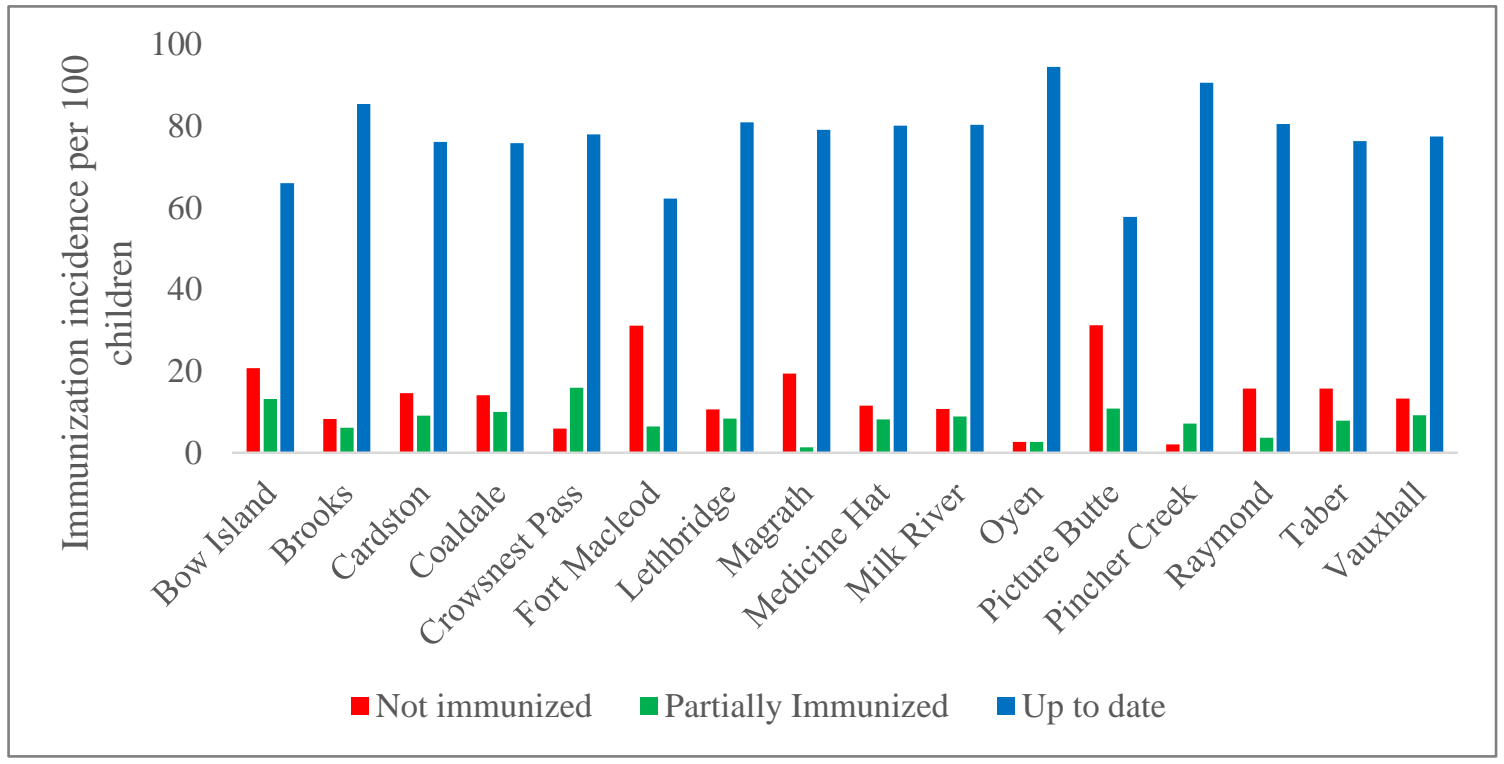

Examining immunization rates by $\mathrm{PHO}$ geographic area, we discovered that more than half of two year olds in Fort Macleod (53.4\%), Picture Butte (64.9\%) and Vauxhall (59.5\%) were unimmunized. The highest incidence rate of children up to date on their vaccinations by age two was in Oyen, at $71.9 \%$, and the majority of full immunization in the PHOs ranged from $56.0 \%$ in Raymond to $69.8 \%$ in Medicine Hat. By age seven, the incidence rate of non-immune children was high in Bow Island (20.8\%), Fort Macleod (31.2\%) and Picture Butte $(31.3 \%)$. These $\mathrm{PHO}$ areas also had low rates of fully immunized seven year old children, at $66.0 \%, 62.3 \%$ and $57.8 \%$ respectively. The incidence rates of fully immunized children were above $90 \%$ in only two PHOs, Pincher Creek (90.7\%) and Oyen (94.6\%). Over $80 \%$ of seven year olds were up to date on their immunizations in Brooks, Lethbridge, Medicine Hat, Milk River and Raymond.

Schools with a seven year old population of five or fewer students were omitted, as was Ralston School in the Medicine Hat $\mathrm{PHO}$, resulting in 80 schools available for analysis. Oyen was removed from analysis as all schools in this $\mathrm{PHO}$ contained fewer than five children aged seven. The mean incidence of partial immunization was 94.4 children per 100 eligible (SD = 11.1), with a median immunization incidence of $100 \%$ and a range of $17 \%$ to $100 \%$. Results for the incidence of fully immunized children were similar, with a mean of $90 \%$ immunization $(S D=12.1)$. The median for fully immunized children in all schools was less than that for partial immunization, at $91 \%$, but the range was the same $(17 \%$ to $100 \%)$. When analyzed by PHO, Fort Macleod $(n=$ 1), Lethbridge $(n=23$, range $=17 \%-100 \%)$, Vauxhall $(n=2$, range $=83 \%-88 \%)$ and Picture Butte $(n=4$, range $=78 \%-100 \%$ ) had the lowest means for full immunization at $83.0 \%, 83.1 \%, 85.5 \%$ and $88.3 \%$ respectively. Results are shown in Figure 3. 
Figure 3. Immunization incidence rates per 100 eligible population by age seven in AHS South Zone public and private schools by PHO catchment area

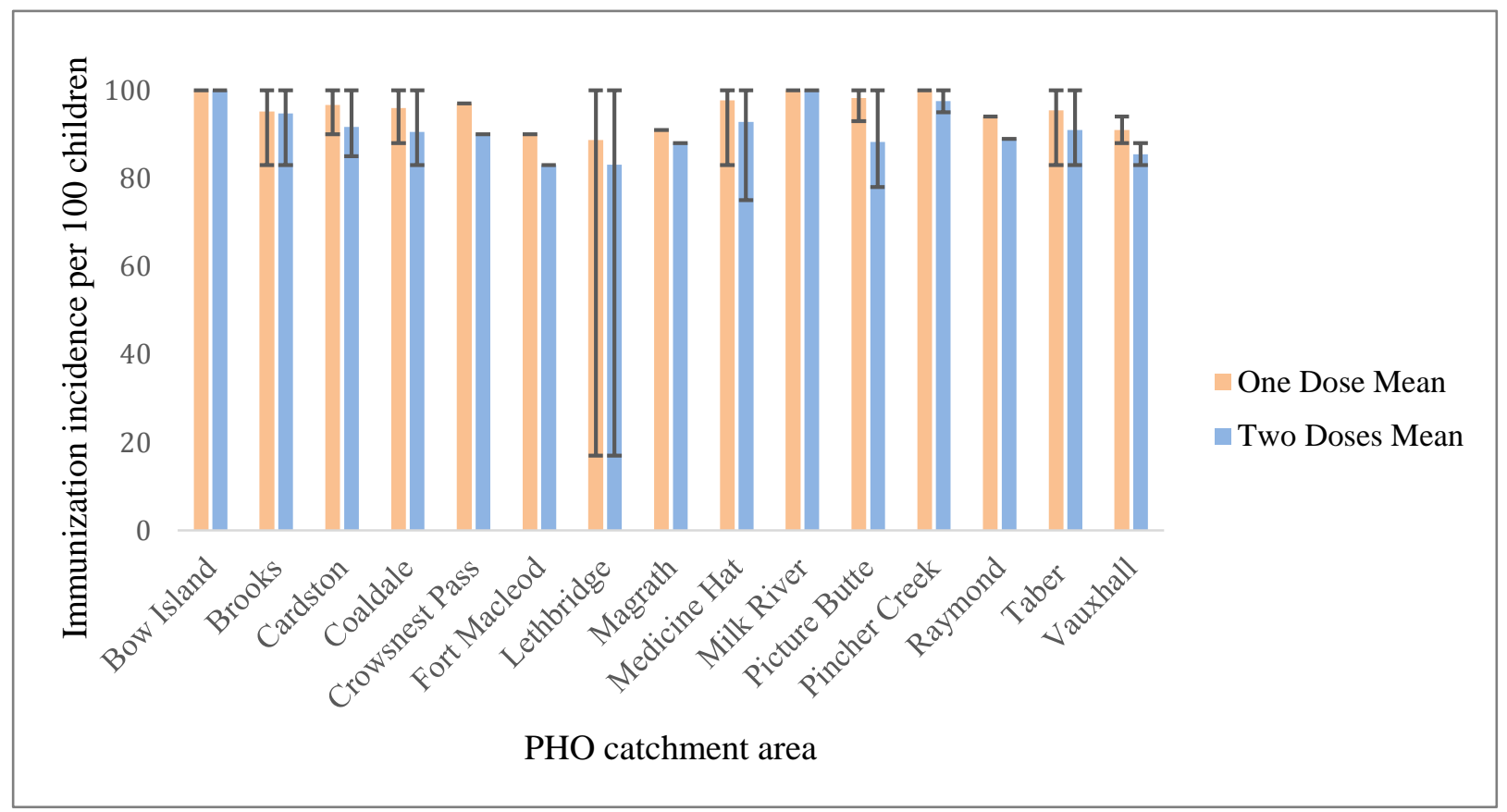

Lines represent mean immunization in schools by $\mathrm{PHO}$, Error bars represent range of immunization status across schools

\section{Discussion}

The results of this study show immunization coverage with measles-containing vaccine to be far below the accepted $90 \%-95 \%$ required for herd immunity in the majority of South Zone communities, and well below the Alberta population targets $(3,5)$. We found that only two communities in southern Alberta had vaccination rates among seven year olds approaching the Alberta Health target of $99 \%$. In further exploration of measles immunization rates at a school level, there is significant heterogeneity among schools for this same 7 year old cohort. School measles immunization rates do not align with the respective community immunization rates. There are two primary reasons for this. First, schools with low immunization rates for measles disease are primarily private schools, where children from specific cultural/religious communities are transported from broad geographic catchment areas by school bus. As an example, the 7 year old immunization rate for the four schools in the Picture Butte area has a range of $78.0 \%$ and median of $87.5 \%$ This community is also one of the locations of residence of children who attend a private school in the Lethbridge PHO catchment area with an immunization rate of $17 \%$ for this age cohort. A second reason to explain the lack of consistency between community and school data is the fact that the community-level data additionally include home-schooled children, who may or may not be immunized. For example, there are a number of Low German Speaking Mennonite home schools where the majority of children are not immunized and where families are quite transient. These schools are not included in the data analysis since public health does not receive school registration lists and does not immunize in these schools.

Awareness of immunization rates by community and by school provides information on the risk of spread of a vaccine-preventable illness such as measles. A school with low immunization rates is a likely place for an outbreak to occur (12). If unimmunized exposed and/or symptomatic children travel from a school to their home communities, measles virus can further spread to vulnerable individuals within these communities. As there are no geographic locations within the South Zone with a high enough immunization rate to confer immunity to the entire population (Figure 3), it becomes increasingly possible for a highly contagious virus such as measles to spread across the South Zone and other parts of the province $(3,13)$. 
This study did not investigate the reasons for low immunization rates at either the community or school level, such as religious beliefs, educational gaps, access to immunization. However, this information could be used in conjunction with previous studies that have explored perspectives of non-immunizing individuals in southern Alberta to inform targeted methods of intervention (8). Furthermore, further research is recommended to identify acceptable methods of school-level intervention for the private schools with low immunization rates. Monitoring of change in immunization rates over time by South Zone community and by school is critical to measuring the impact of interventions.

\section{Conclusion}

There is heterogeneity of immunization uptake for childhood measles-containing vaccine by community and by school in southern Alberta. This study highlights that location of school may not align with geographic community as it pertains to immunization rates. Childhood immunization data analysis at both the community and school level is important in understanding the risks of vaccine-preventable illness spread in a given geographic region such as Alberta South Zone. Data from this study can be used to inform specific interventions required to improve immunization coverage rates in these unique homogeneous communities and their respective schools, and to decrease the risk of measles transmission in southern Alberta.

\section{References}

(1) Moss WJ, Griffin DE. Measles. The Lancet 2012; 379:153-164.

(2) Alberta Immunization Strategy 2007-2017 http://www.health.alberta.ca/documents/ImmunizationStrategy-07.pdf

(3) Rashid H, Khandaker G, Boy R. Vaccination and herd immunity: what more do we know? Curr Opin Infect Dis 2012; 25:243-249.

(4) National Advisory Committee on Immunization. Canadian Immunization Guide: Part 4 - Active Vaccines. Ottawa: Public Health Agency of Canada 2012. http://www.phac-aspc.gc.ca/publicat/cig-gci/p04meas-roug-eng.php

(5) Lievano F, Galea SA, Thornton M, et al. Measles, mumps, and rubella virus vaccine (M-M-R $\left.\mathrm{R}^{\mathrm{TM}} \mathrm{II}\right)$ : a review of 32 years of clinical and postmarketing experience. Vaccine 2012; 30(48):6918-6926.

(6) Leuridan E, Sabbe M, Van Damme P. Measles outbreak in Europe: susceptibility of infants too young to be immunized. Vaccine 2012; 30:5905-5913.

(7) Maltezou HC, Wicker S. Measles in health-care settings. Am J Infect Control 2013; 41:661-663.

(8) Kulig JC, Meyer CJ, Hill SA, Handley CE, Lichtenberger SM, Myck DM. Refusals and delay of immunization within southwest Alberta. Canadian Journal of Public Health 2002: 93(2), 109-112.

(9) Muscat, M. Who gets measles in Europe? J Infect Dis 2011: 204 (Suppl 1), S353-S365

(10) Knol MJ, Urbanus AT, Swart EM, Mollema L, Ruijs WL, van Binnendijk RS, te Wierik MJ, de Melker HE, Timen A, Hahné SJ. Large ongoing measles outbreak in a religious community in the Netherlands since May 2013. Euro Surveill. 2013;18(36):pii=20580.

(11) Alberta Health Services. Routine Immunization Schedule. http://www.health.alberta.ca/healthinfo/imm-routine-schedule.html10.

(12) Lee BR, Feaver SL, Miller CA, Hedberg CW, Ehresmann KR. An elementary school outbreak of varicella attributed to vaccine failure: policy implications. J Infect Dis 2004: 190(3), 477-483.

(13) Parker AA, Staggs W, Dayan GH, et al. Implications of a 2005 measles outbreak in Indiana for sustained elimination of measles in the United States. N Engl J Med 2006; 355:447-455. 


\section{Acknowledgements}

The authors wish to thank Brittany Tyssen, Michelle Davidson and Sjaane Heikoop for their assistance with the analysis of school data and all of the staff from Alberta Health Services and Alberta Health who keep records of immunization status.

\section{Conflict of Interest}

No conflicts of interest to declare.

\section{Funding}

No external funding was received. 